\title{
Phased arrays: A strategy to lower the energy threshold for neu- trinos
}

\author{
Stephanie Wissel ${ }^{1, \star}$, Jessica Avva ${ }^{2,3}$, Keith Bechtol ${ }^{4,3}$, Tyler Chesebro ${ }^{5}$, Linda Cremonesi ${ }^{6}$, \\ Anusha Gupta ${ }^{6}$, Andrew Ludwig ${ }^{7,3}$, Wesley Messino ${ }^{8},{\text { Christian } \text { Miki }^{9}, \text { Ryan Nichol }}^{6}$, Eric Oberla ${ }^{3}$, \\ Andrew Romero-Wolf ${ }^{10}$, David Saltzberg ${ }^{5}$, Chandler Schlupf ${ }^{5}$, Nora Shipp ${ }^{11,3}$, Gary Varner ${ }^{9}$, and \\ Abigail Vieregg $7,12,3$ \\ ${ }^{1}$ Physics Dept., California Polytechnic State Univ., San Luis Obispo CA 93407, USA \\ ${ }^{2}$ Dept. of Physics, Univ. of California Berkeley, Berkeley, CA 94720, USA \\ ${ }^{3}$ Kavli Instit. for Cosmological Physics, Univ. of Chicago, Chicago, Illinois 60637, USA \\ ${ }^{4}$ Wisconsin IceCube Particle Astrophysics Center, Univ. of Wisconsin-Madison, Madison, WI 53703, USA \\ ${ }^{5}$ Dept. of Physics and Astronomy, Univ. of California Los Angeles, Los Angeles, CA 90095, USA \\ ${ }^{6}$ Dept. of Physics and Astronomy, Univ. College London, London, United Kingdom \\ ${ }^{7}$ Dept. of Physics, Univ. of Chicago, Chicago, IL 60637, USA \\ ${ }^{8}$ Electrical Engineering Dept., California Polytechnic State Univ., San Luis Obispo, CA 93407, USA \\ ${ }^{9}$ Dept. of Physics and Astronomy, Univ. of Hawaii, Manoa, HI 96822, USA \\ ${ }^{10} \mathrm{Jet}$ Propulsion Laboratory, California Instit. of Technology, Pasadena, CA 91109, USA \\ ${ }^{11}$ Dept. of Astronomy and Astrophysics, Univ. of Chicago, Chicago, IL 60637, USA \\ ${ }^{12}$ Department of Physics, Enrico Fermi Institute, Univ. of Chicago, Chicago, Illinois 60637, USA
}

\begin{abstract}
In-ice radio arrays are optimized for detecting the highest energy, cosmogenic neutrinos expected to be produced though cosmic ray interactions with background photons. However, there are two expected populations of high energy neutrinos: the astrophysical flux observed by IceCube $(\sim 1 \mathrm{PeV})$ and the cosmogenic flux $\left(\sim 10^{17} \mathrm{eV}\right.$ or $100 \mathrm{PeV}$ ). Typical radio arrays employ a noise-riding trigger, which limits their minimum energy threshold based on the background noise temperature of the ice. Phased radio arrays could lower the energy threshold by combining the signals from several channels before triggering, thereby improving the signal-to-noise at the trigger level. Reducing the energy threshold would allow radio experiments to more efficiently overlap with optical Cherenkov neutrino telescopes as well as for more efficient searches for cosmogenic neutrinos. We discuss the proposed technique and prototypical phased arrays deployed in an anechoic chamber and at Greenland's Summit Station.
\end{abstract}

\section{Introduction: The Phased Array Concept}

In-ice radio neutrino experiments are optimized to detect cosmogenic neutrinos [1] produced through the interaction of ultra-high-energy cosmic rays with the cosmic backgrounds at energies above 50 $\mathrm{PeV}$. However, the recent IceCube observations of astrophysical neutrinos with shower energies of a

^e-mail: swissel@calpoly.edu 
few PeV [2-4] demonstrate that radio experiments with lower energy thresholds (10-PeV scale) are desirable.

Typical experiments [5, 6] rely on a coincidence trigger referenced to the local thermal noise. The temperature of the surrounding ice determines the minimum signal strength that generates a trigger. Coherently summing the signals from several antennas in a phased array involves delaying and summing their signals by the geometric delay between antennas from a given incoming direction, a technique known as beam-forming or the phased array technique. Recent studies [7] point out that beamforming can reduce the receiver noise, and therefore, the trigger and energy threshold of radio neutrino detectors. The received voltage from coherently summed, in-phase plane-wave like signals, such as those expected from the Askaryan effect, would add linearly, whereas the voltages from incoherent background noise would add in quadrature. With this technique, one could use low gain antennas, such as dipoles or bicones, to achieve an improvement in signal-to-noise ratio (SNR) that scales with the square-root of the number of antennas employed in the phased array.

\section{Beamforming in an Anechoic Chamber}

In order to validate the phased array technique, we performed several tests in an anechoic chamber using two to three antennas with identical amplifier chains. Several different antennas were used, including folded dipole antennas from Telewave, Inc. (ANT275D and ANT400D), custom broadband (220-500 MHz) dipoles, and broadband bicones and quad-slot antennas developed for the ARA experiment. Signals from the antennas were amplified by $83 \mathrm{~dB}$ using dual-stage amplifiers (MITEQ AFS400100200-10-15P-4 and Mini-Circuits ZKL-1R5 separated by a 3-dB attenuator) that were powered by pairs of bias tees. The signals were filtered using an Mini-circuits NHP-200 and NLP-450 for all antennas except for the broadband dipoles, which were filtered with a Mini-Circuits NLP-600. The individual antenna voltages as well as the summed beam-formed signal were recorded on a Tektronix MSO5204B oscilloscope. The system noise ( $75 \mathrm{~K})$ was measured by recording waveforms from the same amplifier chains, but with terminated inputs. For impulsive beamforming tests (Section 2.2 and Section 2.3, we also split the signals before the oscilloscope and combined them into the third channel on the oscilloscope.

\subsection{Thermal Noise Correlations}

The expected improvement in signal-to-noise ratio going from individual antennas to a phased array of antennas is predicated on the assumption that thermal noise in adjacent antennas is uncorrelated. For completely overlapping antennas, the $250 \mathrm{~K}$ thermal noise radiating from the ice should be perfectly correlated, whereas antennas separated by a wavelength or more should receive completely uncorrelated noise. Smaller separation distances between antennas reduces the length of the array as well as the overall time delays required to phase up the entire array. Optimally spaced antennas are packed as tightly as possible, without introducing a significant correlated noise component.

To determine the minimum allowable spacing between antennas, we recorded noise traces on the oscilloscope for several different spacings between antennas ranging from as close as physically possible to the maximum separation distance allowed by the dimensions of the anechoic chamber $(1.5 \mathrm{~m})$. For all separation distances, the antenna phase centers of the antennas were aligned parallel to their nulls. The setup was comparable to deploying vertically polarized dipole-like antennas as a phased array in a borehole.

Measurements of thermal noise were compared to a simulation of thermal noise for different antenna spacings following the phasor theory of thermal noise from Goodman [8] combined with 

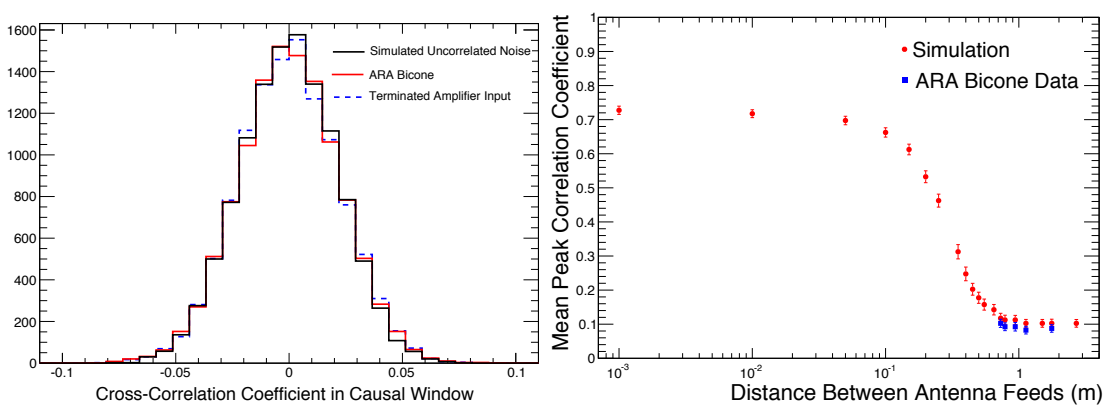

Figure 1. Left: cross-correlation coefficients between two antenna channels spaced $0.73 \mathrm{~m}$ apart over a causal time window \pm 2.4 ns around zero time delay for each of $\sim 500$ recorded events compared to results from terminated amplifiers and the simulation. Right: Mean (points) and standard deviation (error bars) peak crosscorrelation coefficient for 500 simulated or measured events in a $\pm 6.0 \mathrm{~ns}$ time window, which is the causal window for the largest feed-to-feed spacing for which we made measurements $(\sim 2 \mathrm{~m})$.

uncorrelated system noise of 75 K.The left-hand panel of Fig. (1) shows the cross-correlation coefficient in a causal time window normalized by the number of samples and standard deviation of each waveform for the ARA bicones spaced as close as possible, terminated amplifier inputs, and the noise simulation. The measured distribution is consistent with uncorrelated noise, as it matches the distributions for both simulated uncorrelated noise and the uncorrelated system noise of the amplifiers. We observed no significant correlations for measurements at larger separation distances and using other antennas.

Significant noise correlation is only apparent in the simulations at phase-center separation distances that are not physically allowable. The righthand panel of Fig. (1) shows the peak correlation coefficient in a causal window averaged over 500 simulated events and events received on ARA bicones. At small spacings, the correlation coefficient plateaus at 0.75 , corresponding to a fully correlated thermal noise contribution of $300 \mathrm{~K}$ combined with system noise of temperature of $75 \mathrm{~K}$. For physically allowable spacings ( $>0.73 \mathrm{~m}$ ), the simulation overestimates the peak correlation coefficient. We attribute this to imperfect modeling of the antenna and filter phase responses, which would further decorrelate the signals.

\subsection{Analog beam-forming}

The second assumption in the phased array technique is that plane-wave signals add linearly in voltage. We tested this assumption by transmitting broadband, impulsive signals from $4 \mathrm{~m}$ away from the center of a two-antenna phased array of broadband dipoles. The two channels had slightly different impulse responses, gains, and system temperatures due to differences in the antennas and first-stage amplifiers. Due to the differences in the two channels, the beam formed from these two channels should not show the expected $\sqrt{2}$ improvement in SNR expected from identical signal chains.

An analog beam corresponding to an angle broadside to the antennas was formed using a $3 \mathrm{~dB}$ combiner. The SNR averaged over 500 events of the two antennas is $2.6 \sigma$ and $2.7 \sigma$, while the hardware and idealized beam have SNRs of $3.1 \sigma$ and $3.3 \sigma$. The hardware beam matches the idealized beam in SNR, but the signal suffers $12 \%$ loss due to the additional components in the signal chain. 


\subsection{Digital beam-forming}

With a digital phased array, different time delays in firmware or in software can be used to form many beams. Even though beam-forming tends to reduce the overall beam width of an individual beam, the full angular coverage can be recovered by forming multiple beams. The number of independent beams and the individual beam widths are determined by the antenna spacing and the sample rate.

We studied the trigger rate and trigger efficiency of a digital phased array and compared it to a simulation that includes both bandlimited impulses and uncorrelated noise. Using the smallest antennas, the Telewave ANT400D folded dipoles, we mounted three antennas spaced $55 \mathrm{~cm}$ apart on a steel ground mast. An ARA bicone was used as a transmitter along with an Avtech pulser and an attenuator of varying loss. We recorded signals on each antenna at $5 \mathrm{GSa} / \mathrm{s}$ for 500 events at several transmitter attenuations. The signals were then decimated to $1.67 \mathrm{GSa} / \mathrm{s}$ to mimic the Nyquist sampling rate for a system with a bandwidth of $\sim 800 \mathrm{MHz}$. The measured traces were tested against several thresholds to study the rates and efficiency of a digital interferometric trigger.

A trigger is formed when the time-windowed power on each coherently summed beam exceeds a given threshold. The power is calculated for each sample and averaged over the time window. Calculating the power in a wide window (16 samples calculated every 8 samples) reduces the trigger rates for the dispersed pulses in this system, but if the signals were deconvolved first, a shorter sample window may be more efficient. The thresholds-extracted from the simulation due to the limited amount of data recorded in the anechoic chamber-at singles rates of $10,100,1000 \mathrm{~Hz}$, are $2.34 \sigma$, $2.21 \sigma$, and $2.07 \sigma$, respectively.
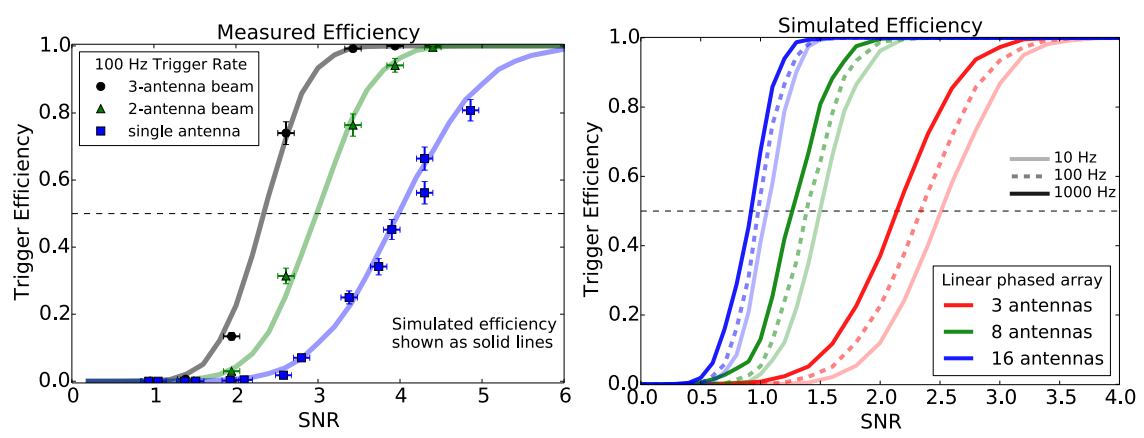

Figure 2. Left: Trigger efficiency vs. SNR in the anechoic chamber measurements (points) compared to a simulation of uncorrelated noise added to the system impulse response (lines). Right: Simulated trigger efficiency for larger phased arrays.

The trigger efficiency for a given input SNR ( $\frac{V_{p k 2 p k}}{2 \sigma}$ on a single antenna) is shown in Fig. (2). Adding additional antennas reduces the $50 \%$ point of the trigger efficiency curves from four for a single antenna down to $2.21 \sigma$ at a fixed trigger rate of $100 \mathrm{~Hz}$. The expected trigger efficiency extrapolated from the simulation of a 16-antenna phased array reduces the trigger threshold to about one.

\section{In-Ice Prototype in Greenland}

In moving from the idealized environment of antennas in air in an anechoic chamber to a phased array deployed in the ice, several factors are of concern. The ice properties and radio backgrounds local to 
the site may be problematic. The length and size of the antenna array may also present a challenge for deployment. To address some of these concerns, we conducted two tests in in the ice at Summit Station in Greenland in both June 2013 [9] and June 2015 [10].

With the lower trigger threshold expected from a phased array, the total visible ice volume at low energies will increase. However, this will have a minimal effect if the propagation loss of the electric field is high. The average field attenuation length of the ice at Summit Station is $\left\langle L_{\alpha}\right\rangle=1022_{-253}^{+230} \mathrm{~m}$. The measured attenuation length was extrapolated to a frequency of $300 \mathrm{MHz}$ from $75 \mathrm{MHz}$ for comparison with other proposed radio neutrino experiments and averaged over the top $1500 \mathrm{~m}$ of ice relevant for neutrino telescopes [9]. The total loss in the electric field strength when combines the losses due to attenuation in the medium with the path loss is shown for three proposed sites in Fig. (3). At likely distances for neutrino events - a few hundred meters - the electric field loss at Summit Station is comparable to the South Pole [12] and lower than at the Ross Ice Shelf [13].

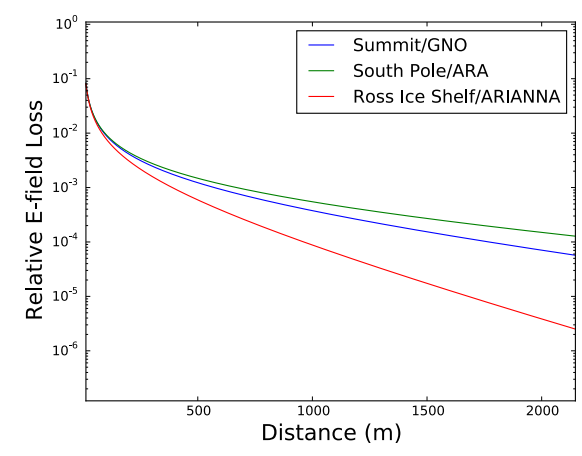

Figure 3. Electric field loss at $300 \mathrm{MHz}$ due to the attenuation of the ice and path loss for potential neutrino detector sites.

During the June 2015 test, a prototypical phased array system, which is described in more detail in Ref. [10], was deployed to characterize the radio backgrounds. The system included 8 broadband dipoles, arranged vertically and separated by $76 \mathrm{~cm}$ feed-to-feed. The bottom of the array was $96 \mathrm{~m}$ below the surface of the ice. RF signals from the antennas were carried along 100-m lengths of coaxial Times Microwave LMR-240 along with DC power for the dual stage amplifier chains described in Section 2 mounted between the antennas in the borehole. Signals from each antenna were split into a beamformer chain and a digitizing chain. The beamformer chain further split each antenna signal eight ways. Eight beams at fixed angles from the horizon were formed using the split antenna channels and fixed delay lines of Times Microwave LMR-200. Traces from both the antenna and beam channels were digitized on four DRS4 evaluation boards that were triggered by a Tektronix MSO5204 oscilloscope. The global trigger provided by the oscilloscope was formed from either automatic (unbiased) or threshold crossing triggers on either an antenna or beam channel.

The improved trigger efficiency found in Section 2.3 assumes that the background noise is uncorrelated thermal noise. The noise averaged over 200 power spectra of 500-sample waveforms observed a downhole antenna, corrected for the overall gain in the system is shown in on the left in Fig. (4). The systematic uncertainty on the absolute scale reflects the uncertainties in the temperature of the cables and amplifiers in the borehole. The observed power is consistent with the expectation from thermal noise in the band between 160 and $440 \mathrm{MHz}$, the $3 \mathrm{~dB}$ points of the filters used in the system.

If the noise in adjacent antennas is uncorrelated, then the RMS voltages in a beamformed channel would be the quadrature sum of the RMS voltages in the individual channels after correcting for the additional loss in the beamformer (-19.9 dB due to the 8-way splitters and 8-way combiners). The RMS voltages of a beam of unbiased triggered events recorded over the course of one night are shown in the righthand panel of Fig. (4). The RMS voltages lie within $10 \%$ difference of the expectation for 
an incoherent signal, and are inconsistent a coherent sum of the signals. In agreement with our results from the anechoic chamber measurements, the noise observed on the downhole beams is incoherent and uncorrelated.
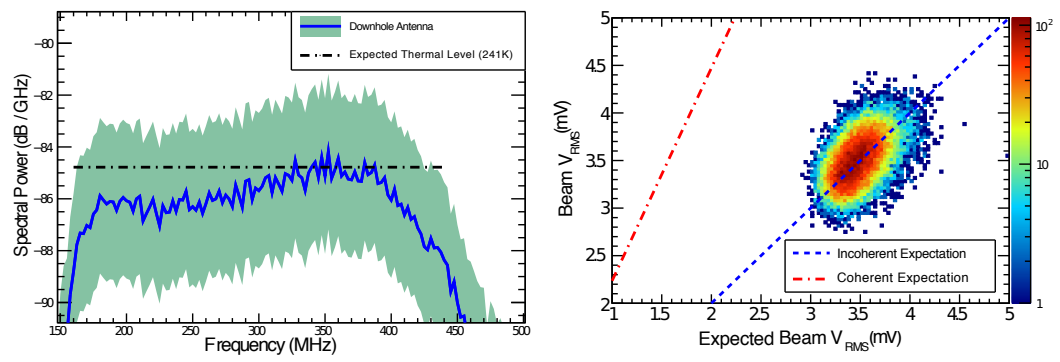

Figure 4. Left: Average power spectral density of thermal-noise triggers of a downhole antenna (blue solid line) and $\pm 3 \mathrm{~dB}$ systematic uncertainty (green band) compared with thermal noise (dashed line). Right: Measured $V_{r m s}$ in a beam compared with the expected RMS voltage for the sum of five borehole antennas.

\section{Conclusions}

The phased array technique relies on two basic assumptions: that a plane-wave like signal adds coherently and the background noise in the antennas adds incoherently. We observed the expected increase in SNR on beams formed in an anechoic chamber. Measurements in an anechoic chamber and at a borehole in Summit Station demonstrate that closely packed antennas aligned along their nulls receive uncorrelated thermal noise.

Triggering with a phased array of low gain antennas shows promise for lowering the energy threshold of in-ice radio neutrino experiments. Using data from the anechoic chamber to inform a simulation that includes realistic parameters for an FPGA, we observe a substantial reduction in trigger threshold when compared to a single-antenna threshold. Such a trigger will be implemented at the South Pole on the ARA experiment, potentially lowering ARA's energy threshold to provide overlap in sensitivity with IceCube.

\section{References}

[1] V.S. Beresinsky, G.T. Zatsepin, Physics Letters B 28, 423 (1969)

[2] IceCube Collaboration, M.G. Aartsen et al., Phys. Rev. Lett. 113, 101101 (2014)

[3] IceCube Collaboration, M.G. Aartsen et al., Phys. Rev. Lett. 111, 021103 (2013)

[4] IceCube Collaboration, M.G. Aartsen et al., Astrophys. J. 809, 98 (2015)

[5] ARA Collaboration, P. Allison et al., Phys. Rev. D93, 082003 (2016)

[6] S.W. Barwick et al., Astroparticle Physics 70, 12 (2015)

[7] A.G. Vieregg, K. Bechtol, A. Romero-Wolf, J. Cosm. and Astropart. Phys. 2, 005 (2016)

[8] J.W. Goodman, Statistical Optics, 2nd Ed. (John Wiley and Sons, Inc., 2015)

[9] J. Avva et al., J. Glaciol. 61, 1005 (2015)

[10] S. Wissel et al., Proc. of Science ICRC2015, 1150 (2015)

[11] R.B. Alley, B.R. Koci, Annals of Glaciology 10 (1988)

[12] T. Barrella, S. Barwick, D. Saltzberg, J. Glaciol. 57, 61 (2011)

[13] J.C. Hanson et al., J. Glaciol. 61 (2015) 